\title{
Erratum: Diabatic-ramping spectroscopy of many-body excited states [Phys. Rev. A 90, 062334 (2014)]
}

\author{
Bryce Yoshimura, W. C. Campbell, and J. K. Freericks
}

(Received 12 September 2017; published 27 September 2017)

DOI: 10.1103/PhysRevA.96.039902

We discovered an error in the units of time in the paper. The time units need to be multiplied by $N$, the number of ions in the crystal. Since all results in the paper are for $N=400$, all time values must be multiplied by 400 . The easiest fix for this, is to change the time-axis labels in all relevant figures (listed below) to $t J_{0} / 400$. These corrections affect the following figures: (i) Fig. 4 (the horizontal axis becomes $t J_{0} / 400$, the red curve is for $\tau=4000 / J_{0}$, and the green curve for $\tau=10,000 / J_{0}$ ); (ii) Fig. $6\left(\tau_{d} J_{0}=4000\right.$ in the label); (iii) Fig. 7 [the horizontal axis of Figs. 7(b) and 7(c) and the labels become $\tau_{d} J_{0}=4000$ ]; (iv) Fig. 9 (labels $\tau_{\text {ramp }} J_{0}$ are equal to 400 for black, 800 for red, and 1600 for green, and also in the caption); and (v) Figs. 10 , 11 , and 13 (the horizontal axis becomes $t_{\text {meas }} J_{0} / 400$ ). In addition, the following changes need to be made in the text: Five lines below Eq. (14), the sentences should read, "We used decoherence times of $\tau_{d} J_{0}=10000$ and 4000 in Fig. 4(a). The total time during our simulations is approximately $32 \mathrm{~ms}$, this is with $J_{0}=2 \pi \times 1.6 \mathrm{kHz}$." In Sec. III, the first paragraph discusses $\tau_{\text {ramp }} J_{0}$ values, which need to be multiplied by 400 to be 1600 and 800 . Also, the second sentence of the first paragraph should read, "We used $J_{0}=2 \pi \times 1.6 \mathrm{kHz}$." On page 10 , the first paragraph of simulated data discusses $\tau_{d} J_{0}$ values, which must be multiplied by 400 to become 10000 and 400 . On page $11, \tau_{d} J_{0}=10000$. These corrections do not change any of our conclusions, but they do make some of the run times a bit long for current state-of-the-art experiments if $J_{0}$ is on the order of $2 \pi \times 1.6 \mathrm{kHz}$. For larger $J_{0}$, this issue becomes less important. 